\title{
Use of Wireless Ultrasound Probe in Isolated Infants: A Case Report of Two SARS-CoV-2-Positive Mothers' Newborns
}

\author{
Giorgia Prontera Alessandro Perri Giovanni Vento Vito D'Andrea \\ Division of Neonatology, Department of Woman and Child Health and Public Health, University Hospital \\ Fondazione Policlinico Gemelli IRCCS, Rome, Italy
}

\section{Estabilished Facts}

- Lung ultrasound has good sensitivity and specificity to identify all the common causes of neonatal distress.

- Point-of-care ultrasound (POCUS) is superior to $\mathrm{x}$-ray evaluation with superior sensitivity and specificity of localizing umbilical catheter tip locations.

- POCUS decreases time to treatment and exposure to radiation for newborns.

\section{Novel Insights}

- The use of wireless probe ultrasound in the diagnosis of neonatal distress syndrome or in umbilical catheter tip location is a safe method to reduce health-care workers' risk of exposure to contagious diseases.

\section{Keywords}

Neonatal intensive care unit - Lung ultrasound · Point-ofcare ultrasound · Respiratory distress syndrome - Umbilical venous catheter $\cdot$ COVID-19 pandemic

\begin{abstract}
The COVID-19 pandemic has upset habits in any workplace. In hospitals, several precautions have been taken to maintain health-care workers' safety and to avoid disease spread or the possible creation of new epidemic outbreaks. The use of medical devices makes the contamination and the nosocomial virus spread possible, causing infection in medical
\end{abstract}

operators and hospitalized patients. In the neonatal intensive care unit, ultrasound has been an increasingly used tool because it is a non-invasive, repeatable method and it is side effect-free as the newborn is not exposed to radiation. It makes a fast diagnosis and then therapy possible such as in the lung diseases and other life-threatening conditions. The use of portable devices such as the wireless probe has many advantages in routine clinical practice, and during the $\mathrm{CO}$ VID-19 pandemic, it has proved to be fundamental for the patient and the physician's safety because it reduced the risk of contamination. We report the use of the wireless ultrasound probe in 2 isolated neonates born to SARS-CoV-2-positive mothers.
Correspondence to:

Vito D’Andrea, dandrea.vito@gmail.com

\section{Karger"}




\section{Introduction}

Ultrasonography (US) has become an essential tool in neonatal diagnosis because it is a non-invasive, bedside, and repeatable method and reduces exposure to radiation in infants [1]. The inclusion of point-of-care ultrasound (POCUS) changed clinical practice, and the clinician tries to answer to a focused question or to achieve a specific procedural goal [2].

Lung ultrasonography (LUS) has become important in the diagnosis of neonatal respiratory diseases, and it has a higher predictive power than chest radiography. It is useful to discriminate between respiratory distress syndrome (RDS) and neonatal transient tachypnoea (TTN) [2]. In RDS, the typical ultrasound signs are pleural line abnormalities, absence of spared areas, and bilateral confluent B-lines. This pattern determines a picture of echographic "white lung" [3]. Instead, in TTN, the interstitial pattern alternates with areas of near-normal lung, and the "double lung point" has been proposed as a pathognomonic finding $[4,5]$. Lung ultrasound is also helpful in detecting life-threatening conditions like pneumothorax and pleural effusion and in providing static guidance for pleurocentesis [6-8].

In RDS, LUS score is accurate and reliable to early identify patients who will need surfactant therapy, allowing for early treatment and reduction of radiation exposure [9].

In the last few years, POCUS has also become increasingly important in other areas such as line placement.

Umbilical venous catheter (UVC) is one of the most used central venous accesses in NICU because it provides safe vascular access immediately after birth in high-risk newborns, and it is used for intravenous administration of parenteral nutrition and drugs and for blood sampling.

The UVC tip position that is considered to be safe to avoid complications is the junction of inferior vena cava and right atrium [10-12]. Thoracoabdominal radiograph is widely used to locate the catheter tip, but over the past few years, ultrasonography has been suggested as the gold standard technique, being more reliable and faster and decreasing radiation and number of manipulations. It also allows the operator to monitor the UVC tip position over the days following insertion, since tip migration frequently occurs [13].

In the last period, during the COVID-19 pandemic, the use of the wireless ultrasound probe has been particularly useful and safe in the management of isolated patients [14]. We report the cases of 2 newborns in whom timely diagnosis of RDS in one and intraprocedural UVC
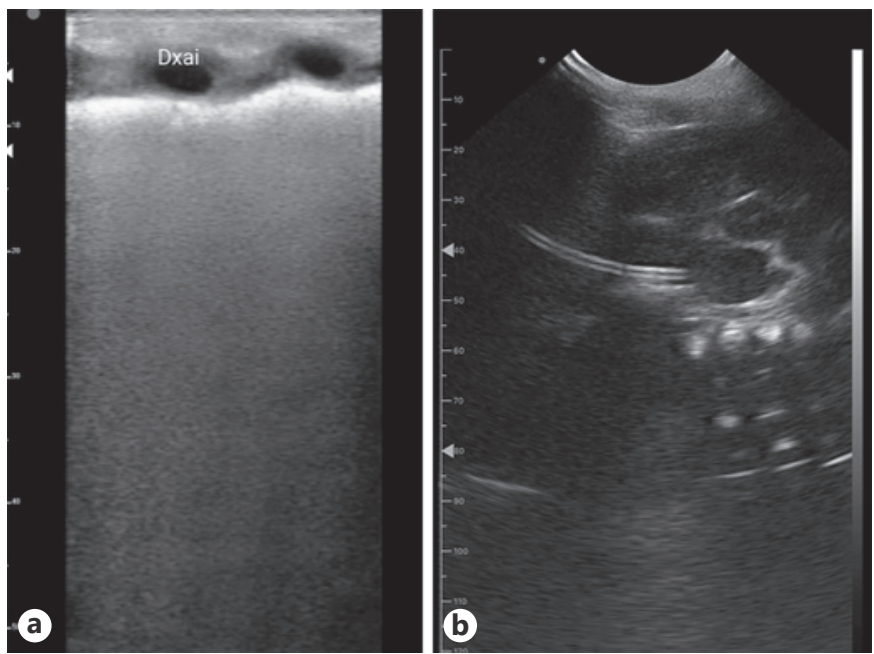

Fig. 1. a Image of white lung in the isolated preterm infant. b U1trasound scan of the correct UVC tip position at the junction of the IVC-RA. IVC, inferior vena cava; RA, right atrium.

tip location in both allowed time-saving management of the infants and, at the same time, safe management for the operators.

\section{Case Report}

\section{Case 1}

A newborn female was born, at $30+1$ weeks of gestational age (birth weight: 1,680 g; APGAR score: $1^{\prime}: 6,5^{\prime}: 8$ ), by urgent caesarean section because of worsening of the respiratory syndrome to a SARS$\mathrm{CoV}$-2-positive mother. At birth she was resuscitated with positive pressure ventilation and then, after stabilization, she was transported in the NICU and placed in an isolated negative pressure room. She was connected to the mechanical ventilator and started on nasal continuous positive air pressure, $6 \mathrm{~cm} \mathrm{H}_{2} \mathrm{O}$, with oxygen requirement progressively increased up to $50 \%$. Therefore, lung ultrasound, which showed a white lung pattern with an LUS score of 12 (Fig. 1a), was performed. A 3.5-Fr single-lumen UVC was then positioned. The first operator was a senior resident; she inserted the UVC and positioned the wireless probe in the sub-xiphoid area. The second operator was a consultant; he handled the picture on the smartphone screen outside the isolation room, and when the catheter tip was seen in the junction of the inferior vena cava and right atrium, the UVC was fixed, $8 \mathrm{~cm}$ from the cutaneous plane. Then infusion of total parental nutrition was started, and endotracheal surfactant was administered using the INSURE technique.

Case 2

A newborn male was born, at 34 weeks of gestational age (birth weight: $1,280 \mathrm{~g}$ [first \%ile]; APGAR score: $\left.1^{\prime}: 3,5^{\prime}: 9\right)$, by caesarean section, due to severe foetal growth restriction, to a SARS-CoV2-positive mother. At birth, ventilation was required for the first minute of life with rapid resumption of heart rate and respiratory 


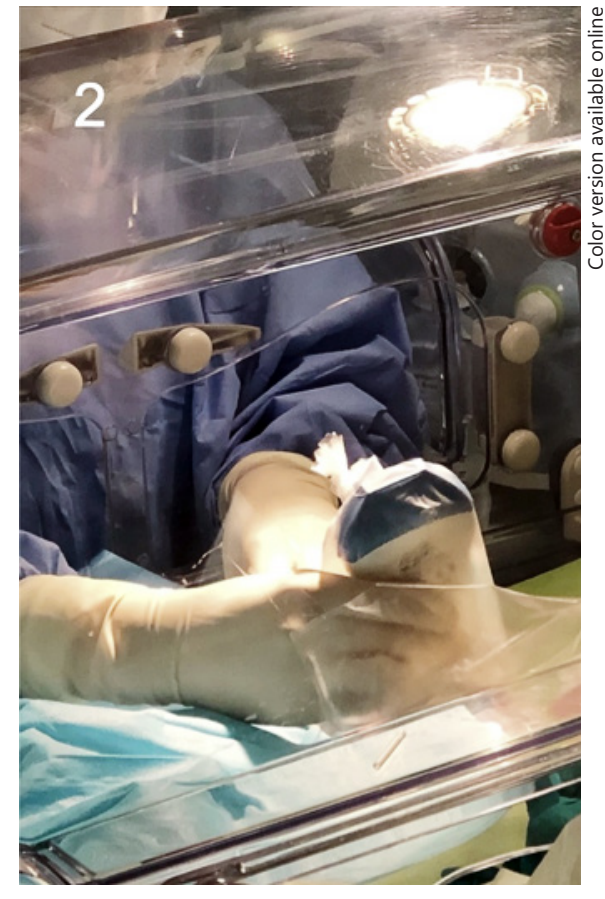

Fig. 2. Wireless ultrasound probe used for tip location of the UVC and lung ultrasound. UVC, umbilical venous catheter.

activity. He was transported in the NICU and placed in an isolated negative pressure room.

After birth prophylaxis and primary care, a 4-Fr single-lumen UVC was positioned. A senior resident inserted the UVC and positioned the wireless probe in the sub-xiphoid area; the second operator, who was the consultant, was located outside the isolation room and visualized on the smartphone screen the picture of the tip in the atrio-caval junction (Fig. 1b). The UVC was then fixed at $8.5 \mathrm{~cm}$ from the cutaneous plane, and infusion of total parental nutrition was started.

\section{Discussion}

In both cases neonatologists, wearing the standard personal protections as per WHO indications [15], used the ultrasound probe and the smartphone which were covered with a sterile single-use plastic cover (Fig. 2). The second operator handled the pictures on the smartphone screen outside the negative pressure room; therefore, he touched neither the patient nor the surrounding materials. The involvement of the trainee in carrying out the ultrasound is interesting. In fact, as a 2020 survey shows, only $7.8 \%$ of residents declared that in their residency school, an official training programme on POCUS exists [16]. The probe used has a double interface Horus (ATL ${ }^{\circledR}$ Milan, Italy): a linear probe for lung ultrasound, using a frequency of $10-12 \mathrm{MHz}$, and a microconvex probe for catheter tip visualization, using a frequency of 5-7 MHz. We performed lung ultrasound observing ESPNIC guidelines [2]; the UVC tip visualization was carried out following the indications of NeoECHOTIP [17]. In the first case, the use of ultrasound with a wireless probe was very useful because it allowed the operators to carry out diagnosis and therapy within the first hour of life of the critically ill newborn. In both cases, the real-time catheter tip location made it possible to safely administer total parenteral nutrition, reducing the waiting time that is usually required when taking $\mathrm{x}$-rays. There are many perks of wireless usage: the probe can be connected to a smartphone or tablet, and therefore the images can be viewed even in another room; it is easily transportable; it turns on quickly; and it has no cables, so accidental infectious contamination is reduced. In addition, after performing the ultrasound, the probe has to be adequately disinfected as per protocol [15].

In conclusion, a wireless ultrasound probe offers maximum safety in the diagnostic management of isolated neonatal patients because it can be used in a sterile environment and for remote diagnosis. Further advantages include ease of use by any medical and paramedical operator, and saving time and cost for treatment.

\section{Statement of Ethics}

This case report is part of a larger study that was approved by the Ethics Committee of the Fondazione Policlinico Universitario A. Gemelli IRCCS, Rome, Italy (ID 3104). Written informed consents were obtained from the parents of all patients for publication of this case report and any accompanying images.

\section{Conflict of Interest Statement}

The authors have no conflicts of interest to declare.

\section{Funding Sources}

No funding was received.

\section{Author Contributions}

G.P. and V.D. idealized equally the study. A.P. and G.V. contributed equally to the drafting of the text.

\section{Data Availability Statement}

All data generated or analysed during this study are included in this article. 


\section{References}

1 Raimondi F, Cattarossi L, Copetti R. Point-ofcare chest ultrasound in the neonatal intensive care unit: an Italian perspective. Neoreviews. 2014 Jan 2;15:e2-6.

2 Singh Y, Tissot C, Fraga MV, Yousef N, Cortes RG, Lopez J, et al. International evidencebased guidelines on point of care ultrasound (POCUS) for critically ill neonates and children issued by the POCUS Working Group of the European Society of Paediatric and Neonatal Intensive Care (ESPNIC). Crit Care. 2020 Feb 24;24(1):65.

3 Brat R, Yousef N, Klifa R, Reynaud S, Shankar Aguilera S, De Luca D. Lung ultrasonography score to evaluate oxygenation and surfactant need in neonates treated with continuous positive airway pressure. JAMA Pediatr. 2015 Aug;169(8):e151797.

4 Copetti R, Cattarossi L. The "double lung point": an ultrasound sign diagnostic of transient tachypnea of the newborn. Neonatology. 2007;91(3):203-9.

5 Raimondi F, Migliaro F, De Luca D, Yousef N, Rodriguez Fanjul J. Clinical data are essential to validate lung ultrasound. Chest. 2016 Jun; 149(6):1575.

6 Cattarossi L, Copetti R, Brusa G, Pintaldi S. Lung ultrasound diagnostic accuracy in neonatal pneumothorax. Can Respir J. 2016;2016: 6515069.
7 Raimondi F, Rodriguez Fanjul J, Aversa S, Chirico G, Yousef N, De Luca D, et al. Lung ultrasound for diagnosing pneumothorax in the critically ill neonate. J Pediatr. 2016 Aug; 175:74-8.e1.

8 Liu J, Chi JH, Ren XL, Li J, Chen YJ, Lu ZL, et al. Lung ultrasonography to diagnose pneumothorax of the newborn. Am J Emerg Med. 2017 Sep;35(9):1298-302.

9 Perri A, Riccardi R, Iannotta R, Di Molfetta DV, Arena R, Vento G, et al. Lung ultrasonography score versus chest $\mathrm{X}$-ray score to predict surfactant administration in newborns with respiratory distress syndrome. Pediatr Pulmonol. 2018 Sep;53(9):1231-6.

10 Anderson J, Leonard D, Braner DA, Lai S, Tegtmeyer K. Videos in clinical medicine. Umbilical vascular catheterization. $\mathrm{N}$ Engl J Med. 2008 Oct 9;359(15):e18.

11 Shahid S, Dutta S, Symington A, Shivananda S, McMaster University N. Standardizing umbilical catheter usage in preterm infants. Pediatrics. 2014 Jun;133(6):e1742-52.
12 Gorski LA, Hadaway L, Hagle ME, Broadhurst D, Clare S, Kleidon T, et al. Infusion therapy standards of practice, 8th edition. J Infus Nurs. 2021 Jan-Feb 1;44(1S Suppl 1): S1-224.

13 Katheria AC, Fleming SE, Kim JH. A randomized controlled trial of ultrasound-guided peripherally inserted central catheters compared with standard radiograph in neonates. J Perinatol. 2013 Oct;33(10):791-4.

14 Buonsenso D, Pata D, Chiaretti A. COVID-19 outbreak: less stethoscope, more ultrasound. Lancet Respir Med. 2020 May;8(5):e27.

15 WHO. Medical devices. Personal protective equipment. Available from: https://www. who.int/teams/health-product-policy-andstandards/assistive-and-medical-technology/ medical-devices/ppe/ppe-covid.

16 Buonsenso D, Malamisura M, Musolino AM. Point-of-care ultrasound training programs across Italian pediatric residency schools. A national survey. Pediatr Emerg Care. 2020 May 19. Epub ahead of print.

17 Barone G, Pittiruti M, Biasucci DG, Elisei D, Iacobone E, La Greca A, et al. Neo-ECHOTIP: a structured protocol for ultrasound-based tip navigation and tip location during placement of central venous access devices in neonates. J Vasc Access. 2021 Apr 5. Epub ahead of print. 